James Serrin

\title{
A maximum principle for mean-curvature type elliptic inequalities
}

Dedicated to Antonio Ambrosetti on the occasion of his sixtieth birthday

Received October 26, 2005

Abstract. Consider the divergence structure elliptic inequality

$$
\operatorname{div}\{\boldsymbol{A}(x, u, D u)\}+B(x, u, D u) \geq 0
$$

in a bounded domain $\Omega \subset \mathbb{R}^{n}$. Here

$$
\boldsymbol{A}(x, z, \boldsymbol{\xi}): K \rightarrow \mathbb{R}^{n}, \quad B(x, z, \boldsymbol{\xi}): K \rightarrow \mathbb{R}, \quad K=\Omega \times \mathbb{R}^{+} \times \mathbb{R}^{n},
$$

and $\boldsymbol{A}, B$ satisfy the following conditions:

$$
\langle\boldsymbol{A}(\boldsymbol{\xi}), \boldsymbol{\xi}\rangle \geq|\xi|-c(x) z-a(x), \quad|\boldsymbol{A}(x, z, \boldsymbol{\xi})| \leq \mathrm{const}, \quad B(x, z, \boldsymbol{\xi}) \leq b(x),
$$

for all $(x, z, \boldsymbol{\xi})) \in K$, where $a(x), b(x), c(x)$ are given non-negative functions. Our interest is in the validity of the maximum principle for solutions of (1), that is, the statement that any solution which satisfies $u \leq 0$ on $\partial \Omega$ must be a priori bounded above in $\Omega$.

This question arises, in particular, when one is interested in the mean curvature equation

$$
\operatorname{div} \frac{D u}{\sqrt{1+|D u|^{2}}}=n H(x) \text {. }
$$

Keywords. Quasilinear elliptic inequalities, maximum principles, mean curvature equation

\section{Introduction}

We are concerned with the divergence structure elliptic inequality

$$
\operatorname{div}\{\boldsymbol{A}(x, u, D u)\}+B(x, u, D u) \geq 0
$$

in a bounded domain $\Omega \subset \mathbb{R}^{n}$. Here

$$
\boldsymbol{A}(x, z, \boldsymbol{\xi}): K \rightarrow \mathbb{R}^{n}, \quad B(x, z, \boldsymbol{\xi}): K \rightarrow \mathbb{R}, \quad K=\Omega \times \mathbb{R}^{+} \times \mathbb{R}^{n},
$$

and $A, B$ satisfy the following structure conditions:

$$
|\boldsymbol{A}(x, z, \boldsymbol{\xi})| \leq \mathrm{const}, \quad\langle\boldsymbol{A}(\boldsymbol{\xi}), \boldsymbol{\xi}\rangle \geq|\xi|-c(x) z-a(x), \quad B(x, z, \boldsymbol{\xi}) \leq b(x),
$$

J. Serrin: Department of Mathematics, University of Minnesota, Minneapolis, MN, USA; e-mail: serrin@math.umn.edu

Mathematics Subject Classification (2000): Primary 35J15; Secondary 35 J70 
for all $(x, z, \boldsymbol{\xi})) \in K$, where $a(x), b(x), c(x)$ are given non-negative functions. Our interest is in the validity of the maximum principle for solutions of (1), that is, the statement that any solution of (1) which satisfies $u \leq 0$ on $\partial \Omega$ must be a priori bounded above in $\Omega$.

This question arises, in the first instance, from the example of the mean curvature inequality

$$
\operatorname{div} \frac{D u}{\sqrt{1+|D u|^{2}}} \geq n H(x),
$$

and secondly from a result of Gilbarg and Trudinger ([1, Theorem 10.10]) for the more general case of inequality (1). In particular, Gilbarg and Trudinger prove the following result.

Let $u \in C(\bar{\Omega}) \cap C^{1}(\Omega)$ be a distribution solution of the inequality $(1)$, where $A, B$ satisfy conditions (2) with $a(x), b(x), c(x)$ constants, written simply $a, b, c .1$ Suppose $u \leq 0$ on $\partial \Omega$ and that

$$
(b+c)|\Omega|^{1 / n}<1 / S
$$

where $S=1 / n \omega_{n}^{1 / n}$ is the Sobolev constant for the space $W_{0}^{1,1}\left(\mathbb{R}^{n}\right)$ and $\omega_{n}$ denotes the measure of the unit ball in $\mathbb{R}^{n}$. Then

$$
u \leq C a \quad \text { in } \Omega \text {, }
$$

with $C$ a constant depending only on $n, b, c$ and $|\Omega|$.

This result can be applied immediately to the mean curvature equation, though for purposes of comparison with our results, we shall defer this until later.

Gilbarg and Trudinger assert as well (without proof) that the same conclusion holds when $u \in W_{0}^{1,1}(\Omega)$ and the coefficients $a(x), b(x), c(x)$ in (2) are in $L^{n}(\Omega)$. It seems to me, however, that the space $L^{n}(\Omega)$ is too weak and should be replaced by $L^{q}(\Omega)$ for some $q>n$. In fact, we have the following result.

Theorem 1. Let $u \in W_{\mathrm{loc}}^{1,1}(\Omega)$ be a distribution solution of the inequality 11 where $A, B$ satisfy conditions (2) with $a(x), b(x), c(x)$ in $L^{q}(\Omega)$ for some $q>n$. Then if ${ }^{2}$

$$
\|b+c\|_{q}|\Omega|^{1 / n-1 / q}<1 / S
$$

we have

where (explicitly)

$$
u \leq C\|a\|_{q}|\Omega|^{1 / n-1 / q} / \delta \quad \text { in } \Omega,
$$

$$
C=C_{1}(n)=3 S\left(3\left[\frac{n}{n-1}\right]^{n-1}\right)^{n q /(q-n)}
$$

and $\delta \in(0,1)$ is any constant such that (4) holds with the right side replaced by $(1-\delta) S$.

(For the record, $(n /(n-1))^{n-1}$ has the universal bound $e$ for all $n \geq 2$.)

\footnotetext{
1 Alternatively, $a(x), b(x), c(x)$ can be bounded by constants, which without confusion can equally be called $a, b, c$.

2 By $\|\cdot\|_{q}$ we mean $\|\cdot\|_{L^{q}(\Omega)}$.
} 
If $a(x), b(x), c(x)$ are bounded, the conclusion can be strengthened. It is convenient here to introduce the effective radius $\mathcal{R}$ of $\Omega$ by the definition

$$
|\Omega|=\omega_{n} \mathcal{R}^{n} .
$$

We can then replace (4) by

$$
(b+c) \mathcal{R}<n,
$$

and (5) by

$$
u \leq C a \mathcal{R} / \delta
$$

where now

$$
C=C_{2}(n)=\frac{2}{n}\left(3\left[\frac{n}{n-1}\right]^{n-1}\right)^{n} .
$$

Condition (4) in Theorem 1 can in fact be replaced by the weaker restriction

$$
\int_{\Omega}\left(\frac{b+c}{n}\right)^{n}<\omega_{n},
$$

but at the cost that the conclusion (5) arises in the less precise form

$$
u \leq C\left(n, q,|\Omega| ;\|b+c\|_{n},\|b+c\|_{q}\right)\|a\|_{q},
$$

with no simple expression for $C$ in terms of the listed parameters. Moreover, $C \rightarrow \infty$ not only as $q \rightarrow n$ but also as $\|b+c\|_{q} \rightarrow \infty$, even when $\|b+c\|_{n}$ is finite.

Theorem 1 and the extensions given above will be proved in Section 1 . For the explicit case of the mean curvature inequality $(3$, the conclusions take the following form.

Theorem 2. Let $u \in W_{\mathrm{loc}}^{1,1}(\Omega)$ be a distribution solution of the mean curvature inequality (3), with $u \leq 0$ on $\partial \Omega$. Assume that $H^{-} \in L^{q}(\Omega)$ for some $q>n$. Then if

$$
\left\|H^{-}\right\|_{q}|\Omega|^{1 / n-1 / q}<\omega_{n}^{1 / n}
$$

we have

$$
u \leq C \mathcal{R} / \delta
$$

where

$$
C=C_{3}(n)=\frac{1}{n}\left(3\left[\frac{n}{n-1}\right]^{n-1}\right)^{n q /(q-n)} .
$$

If $H(x)$ is bounded below, say $H(x) \geq-H_{0}, H_{0}>0$, the conclusion can be strengthened, with (9) replaced by

$$
H_{0} \mathcal{R}<1
$$

and 11] by

$$
C=C_{4}(n)=\frac{0.6}{n}\left(3\left[\frac{n}{n-1}\right]^{n-1}\right)^{n} .
$$


Corresponding to condition (7), it is actually enough to assume only that

$$
\left\|H^{-}\right\|_{n}^{n}<\omega_{n},
$$

though the conclusion again takes the less explicit form

$$
u \leq C\left(n, q,|\Omega| ;\|H\|_{n},\|H\|_{q}\right) .
$$

In the canonical case $n=2, H(x)=-H_{0}$ and $\delta=1 / 2$ the estimate $(10$ ) can be replaced by (see Section 3 )

$$
u \leq 15 \mathcal{R}
$$

Of course this is not too accurate for the case of balls, or even convex sets, where optimal estimates can be obtained, at least for $C^{1}$ solutions, by using the standard maximum principle and spherical caps as comparison functions. See also the comments in Section 3

Theorem 2 appears to be new. We emphasize in particular that the solution class consists of weakly differentiable functions, that $\Omega$ is unrestricted except to have finite measure, and finally that $(11),(11)$ ) and $(14)$ are all explicit.

It remains an open question, at least to the present author, whether the space $L^{q}(\Omega)$ for the coefficients can be replaced by $L^{n}(\Omega)$, as asserted by Gilbarg and Trudinger (see the comments at the foot of page 276 and the top of page 277 of [1]). This is, in fact, true when the solution is in the strong class $C^{2}(\Omega)$ (or even $W_{\text {loc }}^{2, n}(\Omega)$ ), as proved by the same authors (Corollary 10.6), but again it is an open question whether the smoothness condition can be removed.

The reader may question whether the restrictions (9) or (9) are necessary for the validity of (10). In fact, for the specific case $H(x) \geq-H_{0}=$ negative constant, no solution at all is possible if $\Omega$ contains a ball $B=B_{R}$ of radius $R$ and $H_{0} \geq 1 / R$. To see this, take a hemispherical cap $v=v(x)$ over the ball, of radius exactly $R$. The graph $z=v(x)$ over $B$ has mean curvature $-1 / R$, so that

$$
\operatorname{div}\left(\frac{D v}{\sqrt{1+|D v|^{2}}}\right)+n / R=0 \quad \text { in } B .
$$

By a vertical translation of $v(x)$, either up or down, it can be arranged that the cap is tangent to the graph $z=u(x)$ at some point $x_{0} \in B$, while also $v(x) \leq u(x)$ in $B$. The translated cap of course continues to solve (15).

We can now apply a beautiful theorem of Eberhard Hopf ([2, Satz 3']; see also [3, Theorem 2.5]) concerning tangent solutions of quasilinear elliptic equations, to conclude that in this case $u \equiv v$ in $B$, which is obviously impossible.

A simple consequence is that if $\Omega$ is a ball of radius $R$ and $H(x)=$ constant $=-H$, then the condition $H R \leq 1$ is necessary for the mere existence of a solution, while $H R<1$ is sufficient to provide an upper bound on the solution in terms of its boundary values.

Section 1 is devoted to the proof of Theorem 1 , and Section 2 to the proof of Theorem 2 In Section 3 we add some final remarks. 


\section{Proof of Theorem 1}

For completeness we first recall that a function $u \in L_{\mathrm{loc}}^{1}(\Omega)$ is a weak distribution solution of 11 in $\Omega$ if $\boldsymbol{A}(\cdot, u, D u)$ is measurable in $\Omega ; B(\cdot, u, D u) \in L_{\mathrm{loc}}^{1}(\Omega)$; and

$$
\int_{\Omega}\langle\boldsymbol{A}(x, u, D u), D \varphi\rangle \leq \int_{\Omega} B(x, u, D u) \varphi
$$

for all $\varphi \in C^{1}(\Omega)$ such that $\varphi \geq 0$ in $\Omega$ and $\varphi \equiv 0$ near $\partial \Omega$. (In view of the condition $|\boldsymbol{A}| \leq$ const in $(2)$, this inequality is meaningful.)

In order to have a sufficiently large supply of test functions $\varphi$ for later purposes, the following lemma is crucial.

Lemma 1. Let $\psi: \mathbb{R} \rightarrow \mathbb{R}_{0}^{+}$be a non-decreasing continuous function such that $\psi(t)=0$ for $t \in(-\infty, \ell]$ and $\psi \in C^{1}[\ell, m] \cap C^{1}[m, \infty)$, with possible corners at $t=\ell$ and $t=m, \ell \leq m$, and with $\psi^{\prime}$ uniformly bounded. If $u \in W_{\mathrm{loc}}^{1,1}(\Omega)$ is a distribution solution of (1) such that $u \leq 0$ on $\partial \Omega$, then (16) is valid for $\varphi=\psi(u)$, in the sense that

$$
\int_{\Omega}\langle\boldsymbol{A}(x, u, D u), D \varphi\rangle \leq \int_{\Omega}[B(x, u, D u)]^{+} \varphi .
$$

A proof can be found in [4, Section 3.1].

The proof of Theorem 1 is based on two main lemmas.

Lemma 2. Let $w \in W_{\mathrm{loc}}^{1,1}(\Omega)$ be a solution of inequality $(1$, and suppose the coefficients $a(x), b(x), c(x)$ in $(2)$ are in the Lebesgue space $L^{q}(\Omega)$ for some $q>n$. Assume that $|\Omega|=1$ and

$$
w \geq k \quad \text { in } \Omega, \quad w=k \quad \text { on } \partial \Omega,
$$

with $k=\alpha S\|a\|_{q}, \alpha>0$ and $S=1 / n \omega_{n}^{1 / n}$. Then $w \in L^{\infty}(\Omega)$ and

$$
w \leq\left(K\left[\frac{n}{n-1}\right]^{n-1}\right)^{n q /(q-n)}\|w\|_{1}, \quad K=e^{1 / e}+1 / \alpha+S\|b+c\|_{q} .
$$

(In the case of bounded coefficients we can take more simply $K=1+1 / \alpha+S(b+c)$.)

Proof. Step 1. This relies on a Moser-type iteration argument. We argue by induction, showing that if $w \in W_{\text {loc }}^{1,1}(\Omega) \cap L^{r}(\Omega)$ for some $r \geq 1$, then

$$
w \in W_{\mathrm{loc}}^{1,1}(\Omega) \cap L^{\kappa r}(\Omega),
$$

where $\kappa=n /(n-1)$. For $r=1$ one obviously has $w \in W_{\text {loc }}^{1,1}(\Omega) \cap L^{1}(\Omega)$, since $w=k$ on $\partial \Omega$. Hence by induction

$$
w \in W_{\mathrm{loc}}^{1,1}(\Omega) \cap L^{\varrho}(\Omega) \quad \text { for all } \varrho \geq 1 .
$$


Let $\ell, m$ be fixed, with $k<\ell<m$ (ultimately we take $\ell \rightarrow k$ and $m \rightarrow \infty$ ). For all real numbers $r \geq 1$, define

$$
\begin{aligned}
& \psi(t)= \begin{cases}0 & \text { if } k \leq t \leq \ell, \\
t^{r}-\ell^{r} & \text { if } \ell<t<m, \\
m^{r}-l^{r} & \text { if } t \geq m ;\end{cases} \\
& v(t)= \begin{cases}\ell^{r} & \text { if } k \leq t \leq \ell, \\
t^{r} & \text { if } \ell<t<m, \\
m^{r} & \text { if } t \geq m .\end{cases}
\end{aligned}
$$

The truncation of $\psi(t)$ and $v(t)$ when $t \geq m$ makes $\psi(t)$ and $v(t)$ piecewise smooth with corners only at $t=\ell$ and $t=m$.

By Lemma 1 it is clear that $\varphi=\psi(w)$ can serve as a test function for 11 in $\Omega$. In particular by (17) we have

$$
\int_{\Omega}\langle\boldsymbol{A}(x, w, D w), D \varphi\rangle \leq \int_{\Omega}[B(x, w, D w)]^{+} \varphi,
$$

where $D \varphi=\mathbf{0}$ in $\Omega \backslash \Gamma$ and

$$
D \varphi=r w^{r-1} D w \quad \text { in } \Gamma,
$$

with $\Gamma=\{x \in \Omega: \ell<w(x)<m\}$. For $x \in \Gamma$,

$$
r w^{r-1}|D w|=|D v|, \quad v=v(w)=w^{r} .
$$

Hence by the structure conditions (2), the inequality (21) leads to

$$
\|D v\|_{1} \leq r \int_{\Omega}(b+c+a / k) v .
$$

where we have used the fact that $(23)$ is trivially satisfied on the set $\Omega \backslash \Gamma$. (The integrals in 23 are well defined, since $v \leq m^{r}$ and $D v \in L^{1}(\Omega)$. Even more, $k>0$ unless $a \equiv 0$.)

Step 2. By Hölder's inequality and the fact that $k=\alpha S\|a\|$ we obtain from 23,

$$
\|D v\|_{1} \leq r\left(\|b+c\|_{q}+1 / \alpha S\right)\|v\|_{1}^{1-n / q}\|v\|_{n /(n-1)}^{n / q} .
$$

On the other hand, by Sobolev's inequality (recall $v \equiv \ell^{r}$ near $\partial \Omega$ ) it follows that $\left\|v-\ell^{r}\right\|_{n /(n-1)} \leq S\|D v\|_{1}$. But also $\left\|\ell^{r}\right\|_{n /(n-1)}=\left\|\ell^{r}\right\|_{1}$ since $|\Omega|=1$. Therefore

$$
\|v\|_{n /(n-1)} \leq\left\|v-\ell^{r}\right\|_{n /(n-1)}+\left\|\ell^{r}\right\|_{n /(n-1)} \leq S\|D v\|_{1}+\left\|\ell^{r}\right\|_{1} \leq S\|D v\|_{1}+\|v\|_{1} .
$$

since $v \geq \ell^{r}$ in $\Omega$. Combining the previous two displayed inequalities now gives

$$
\|v\|_{n /(n-1)} \leq S r d\|v\|_{1}^{1-n / q}\|v\|_{n /(n-1)}^{n / q}+\|v\|_{1},
$$

where $d=\|b+c\|_{q}+1 / \alpha S$. 
Application of Young's inequality with the exponent pair $(q /(q-n), q / n)$ shows that

$$
\operatorname{Srd}\|v\|_{1}^{1-n / q}\|v\|_{n /(n-1)}^{n / q} \leq\left(1-\frac{n}{q}\right)(\operatorname{Sr} d)^{q /(q-n)}\|v\|_{1}+\frac{n}{q}\|v\|_{n /(n-1)} .
$$

Thus (24) implies (!)

$$
\|v\|_{n /(n-1)} \leq\left[(S r d)^{q /(q-n)}+q /(q-n)\right]\|v\|_{1} \leq\left[\operatorname{Srd}+e^{1 / e}\right]^{q /(q-n)}\|v\|_{1} .
$$

Step 3. It is not hard to check that

$$
\left\|w^{r}\right\|_{\kappa, \Gamma^{\prime}} \leq\|v\|_{\kappa}=\|v\|_{n /(n-1)}, \quad \Gamma^{\prime}=\{x \in \Omega: k \leq w(x)<m\}
$$

with $\kappa=n /(n-1)$. Similarly

$$
\|v\|_{1} \leq\left\|w^{r}\right\|_{1}+\left(\ell^{r}-k^{r}\right)|\Omega \backslash \Gamma| \leq\left\|w^{r}\right\|_{1}+\ell^{r}-k^{r} .
$$

Hence the concluding inequality of Step 2 can be rewritten

$$
\left\|w^{r}\right\|_{\kappa, \Gamma^{\prime}} \leq[K r]^{q /(q-n)}\left\{\left\|w^{r}\right\|_{1}+\ell^{r}-k^{r}\right\},
$$

with $K=1 / \alpha+e^{1 / e}+S\|b+c\|_{q}$. Letting $\ell \rightarrow k$ gives $\left\|w^{r}\right\|_{\kappa, \Gamma^{\prime}} \leq[K r]^{q /(q-n)}\left\|w^{r}\right\|_{1}$. Finally, taking $m \rightarrow \infty$ and using the monotone convergence theorem, there follows

$$
\|w\|_{\kappa r}^{r} \leq[K r]^{v}\|w\|_{r}^{r}, \quad v=q /(q-n) .
$$

The right side of 25$]$ is finite by the induction assumption $w \in L^{r}(\Omega)$. Thus also $\|w\|_{\kappa r}$ is finite, completing the induction step and consequently showing that $w \in L^{\varrho}(\Omega)$ for all $\varrho \geq 1$.

Step 4. The inequality (25) holds for all $r \geq 1$. Taking first $r=1$, we get

$$
\|w\|_{\kappa} \leq K^{v}\|w\|_{1}
$$

Next, take $r=\kappa$ so that

$$
\|w\|_{\kappa^{2}} \leq(K \kappa)^{v / \kappa}\|w\|_{\kappa} \leq\left[(K \kappa)^{1 / \kappa} K\right]^{v}\|w\|_{1}=\left[K^{1+1 / \kappa} \kappa^{1 / \kappa}\right]^{v}\|w\|_{1} .
$$

Continuing in this way, with $r$ successively equal to $1, \kappa, \kappa^{2}$, etc., we get

$$
\|w\|_{\kappa^{j}} \leq\left[K^{\Sigma} \kappa^{\Sigma^{\prime}}\right]^{v}\|w\|_{1}
$$

where

$$
\Sigma=\Sigma_{j}=\sum_{i=0}^{j-1} \frac{1}{\kappa^{i}}, \quad \Sigma^{\prime}=\Sigma_{j}^{\prime}=\sum_{i=1}^{j-1} \frac{i}{\kappa^{i}} .
$$

The series $\Sigma$ converges to $\kappa /(\kappa-1)=n$ as $j \rightarrow \infty$, and similarly $\Sigma^{\prime}$ converges to $\kappa /(\kappa-1)^{2}=n(n-1)$. Thus letting $j \rightarrow \infty$ in (26) gives

$$
\|w\|_{\infty} \leq\left(K\left[\frac{n}{n-1}\right]^{n-1}\right)^{n q /(q-n)}\|w\|_{1} .
$$


If $a, b, c$ are bounded, we can effectively suppose $q=\infty$, so that (24) reduces to

$$
\|v\|_{\kappa} \leq[\operatorname{Srd}+1]\|v\|_{1} .
$$

Hence in this case one can take $K=1+1 / \alpha+S(b+c)$. The proof is complete.

Lemma 3. Let the hypotheses of Lemma 2 hold with the exception that

$$
\|b+c\|_{n} \leq(1-\delta) / S
$$

where $0<\delta<1$. Then

$$
\|w\|_{1} \leq(1+\alpha) S\|a\|_{q} / \delta .
$$

Proof. As in the proof of Lemma 2, but using only the case $r=1$, we obtain corresponding to 23,

$$
\|D v\|_{1} \leq \int_{\Omega}[(b+c) v+a] .
$$

In turn, by Hölder's inequality,

$$
\|D v\|_{1} \leq\|b+c\|_{n}\|v\|_{n /(n-1)}+\|a\|_{1} \leq \frac{1-\delta}{S}\|v\|_{n /(n-1)}+\|a\|_{1}
$$

by 28 .

Next by Sobolev's inequality, as before,

$$
\|v\|_{n /(n-1)} \leq\|v-\ell\|_{n /(n-1)}+\|\ell\|_{n /(n-1)} \leq S\|D v\|_{1}+\ell .
$$

Using (30), this gives

$$
\|v\|_{n /(n-1)} \leq(1-\delta)\|v\|_{n /(n-1)}+S\|a\|_{1}+\ell .
$$

After transposing, one can take $\ell \rightarrow k$ and $m \rightarrow \infty$. Then as in the proof of Lemma 2 there follows

$$
\|w\|_{n /(n-1)} \leq(1+\alpha) S\|a\|_{q} / \delta
$$

since $k=\alpha S\|a\|$. The required conclusion (29) is now obvious.

Proof of Theorem 1 . First take $|\Omega|=1$. Define $w=u^{+}+k$. Then $w \geq k$ in $\Omega$ and $w=k$ on $\partial \Omega$, so that Lemmas 2 and 3 apply. In particular by (4) and (18),

$$
K=e^{1 / e}+1 / \alpha+S\|b+c\| \leq e^{1 / e}+1 / \alpha+1-\delta .
$$

We are free to choose $\alpha$ as we wish. For bounded coefficients take, say, $\alpha=1$, and replace $e^{1 / e}$ by 1 . Then $1+\alpha=2$ and $K \leq 2+1 / \alpha=3$. Otherwise take $\alpha=2$, giving $1+\alpha=3$ and $K \leq 3$.

The conclusions $(5)$ and $(5)$ ) for the case $|\Omega|=1$ now follow at once from $(18)$ and 29). The general result is then obtained by scaling.

These estimates can be compared with the case $p=1$ of Theorem 10.10 in [1]. A boundary condition $u \leq M$ on $\partial \Omega$ can be handled by the change of variable $\tilde{u}=u-M$ and replacing $a$ by $a+M c$. 


\section{The mean curvature inequality}

Consider the mean curvature inequality

$$
\operatorname{div}\left(\frac{D u}{\sqrt{1+|D u|^{2}}}\right) \geq n H(x)
$$

Putting $\boldsymbol{A}(\boldsymbol{\xi})=\boldsymbol{\xi} / \sqrt{1+|\boldsymbol{\xi}|^{2}}, B=-n H(x)$, we see that $|\boldsymbol{A}|<1$ and

$$
\langle\boldsymbol{A}(\boldsymbol{\xi}), \boldsymbol{\xi}\rangle \geq|\boldsymbol{\xi}|-a, \quad a=\sqrt{\frac{5 \sqrt{5}-11}{2}} \sim 0.3002831 .
$$

[This is an easy exercise in differential calculus. In fact, the best value for $a$ is

$$
\max _{0 \leq t<\infty}\left(t-t^{2} / \sqrt{1+t^{2}}\right) .
$$

By elementary calculus the maximum occurs at $t_{0}=\sqrt{(\sqrt{5}-1) / 2} \sim 0.78614$, and in turn $t_{0}-t_{0}^{2} / \sqrt{1+t_{0}^{2}}$ takes the value $a$ given above.]

Thus (33) satisfies the structure conditions (2), with $a$ as given, $c=0$ and $b=$ $-n H^{-1}(x)$. From Theorem 1 we then get

$$
u \leq\left(3\left[\frac{n}{n-1}\right]^{n-1}\right)^{n q /(q-n)} S|\Omega|^{1 / n} / \delta
$$

provided $\left\|H^{-}\right\|_{q}|\Omega|^{1 / n-1 / q} \leq(1-\delta) \omega_{n}^{1 / n}$. Rewriting this in terms of the effective radius $\mathcal{R}$ of $\Omega$, it becomes

$$
u \leq \frac{1}{n}\left(3\left[\frac{n}{n-1}\right]^{n-1}\right)^{n q /(q-n)} \mathcal{R} / \delta,
$$

which is just 10 - 11 . In the same way we obtain $10-111)$. This proves Theorem 2.

In the canonical case $n=2, H(x) \geq-H_{0}$ and $\delta=1 / 2$ the estimate (10)-(11) can be somewhat improved. In fact, from (32) we can take $K=3 / 2+1 / \alpha$. Then with $\alpha=1.1$, $(n /(n-1))^{n-1}=2$, we see from 18 , 29) that $u / \mathcal{R}=2.1 \cdot(5.3 / 1.1)^{2} a \sim 14.6$, as required for (14).

Remark. If $u \leq 0$ on $\partial \Omega$ and $H=0$ one expects the conclusion $u \leq 0$ in $\Omega$. This however cannot be obtained by the present approach since the constant $a \sim 0.3002831$ acts as an inhomogeneous term in the structure conditions (2). 


\section{Remarks}

The a priori estimates $\sqrt{10}-(11)$ for the supremum of a solution of $(3)$ in $\Omega$ can be supplemented, as we have noted above, by the result of Corollary 10.6 in [1] as well as by simple bounds based on spherical caps (or even the upper part of cylindrical tubes). Each of these results has its own separate merit, depending on given smoothness assumptions on $u$, or on the geometry of the domain $\Omega$.

A final esimate, obtained also by Gilbarg and Trudinger ([1, p. 409]) can also be noted. In particular, assume that the boundary $\partial \Omega$ of $\Omega$ is of class $C^{2, \alpha}$ (no smoothness conditions on $\partial \Omega$ were required in the previous results). Suppose also that the mean curvature $H^{\prime}$ of the boundary satisfies

$$
H^{\prime}(y) \geq \frac{n}{n-1} H_{0}
$$

where $H(x) \geq-H_{0}=$ negative constant. Then the following upper bound holds.

Let $u$ be a solution of (3) of class $C^{1}(\Omega)$. Then if $u \leq 0$ on $\partial \Omega$ we have

$$
u \leq\left(e^{\mu D}-1\right) / \mu \quad \text { in } \Omega
$$

where $\mu=1+n H_{0}$ and $D=\operatorname{diam} \Omega$.

Gilbarg and Trudinger in fact assume that $H(x) \equiv$ const, that $u \in C^{2}(\Omega)$, and that $u$ solves (3) with equality, but it is not hard to see that their proof implies the more general result stated here.

When $\Omega$ is a ball of radius $R$, condition $(35)$ becomes $1 / R \geq(n /(n-1)) H_{0}$, that is, $H_{0} R \leq(n-1) / n$, a condition somewhat stronger than 9$)$. Since obviously $D=2 R$ for balls, 36 gives

$$
u \leq\left(e^{2\left(1+n H_{0}\right) R}-1\right) /\left(1+n H_{0}\right) .
$$

If $R$ is small the bound is essentially $u \leq 2 R$, which is quite good. On the other hand, for large $R$ clearly $(37)$ is not nearly as good as $(10)$ or $(14)$.

Whatever else, it should be kept in mind (i) that condition 35 is exactly the boundary curvature condition necessary for the existence of solutions of the Dirichlet problem for the constant mean curvature equation for arbitrary continuous data, while (ii) the estimate (36) does not apply at all in the generality of Theorem 1

Acknowledgements. The author wishes to thank Professor Patrizia Pucci for her help and for her kind interest in this work.

\section{References}

[1] Gilbarg, D., Trudinger, N.: Elliptic Partial Differential Equations of Second Order. 2nd ed., Springer (1983) Zbl 0562.35001 MR 0737190

[2] Hopf, E.: Elementare Bemerkungen über die Lösungen partieller Differentialgleichungen zweiter Ordnung vom elliptischen Typus. Sitz. Preussischen Akad. Wissenschaften 1927, $147-$ 152 JFM 53.0454.02

[3] Pucci, P., Serrin, J., The strong maximum principle revisited. J. Differential Equations 196, 1-66 (2004); Erratum, J. Differential Equations 207, 226-227 (2005) Zbl pre02033000MR2100819

[4] Pucci, P., Serrin, J.: The maximum principle. In: Handbook of Differential Equations, M. Chipot and P. Quittner (eds.), Elsevier (2006) 